\title{
A Comparative Study between Brightness Preserving Bi- histogram and Tri-histogram Equalization for Image Contrast Enhancement
}

\author{
Al Mehdi Saadat Chowdhury \\ Lecturer \\ Department of Computer Science and Engineering \\ North East University Bangladesh \\ Bangladesh
}

\author{
M. Shahidur Rahman \\ Professor \\ Department of Computer Science and Engineering \\ Shahjalal University of Science and Technology \\ Bangladesh
}

\begin{abstract}
This paper compares brightness preserving image enhancement techniques using bi-histogram equalization and tri-histogram equalization methods. Traditionally for image contrast enhancement, global histogram equalization technique is used extensively. However, global histogram equalization tends to change the mean brightness of any image to the middle gray level of the dynamic range, which often results in over or under enhancement and introduce some annoying artifacts. To overcome such problems, several bi-histogram based techniques and tri-histogram based technique has been proposed. While bi-histogram based techniques divides the histogram of any image into two subhistograms and equalize them independently, tri-histogram based technique divides the histogram into three sub histograms. This paper compares some of these equalization techniques. Simulation results can be quantitative and qualitative in nature. For quantitative analysis, Absolute Mean Brightness Error (AMBE) measurement has been used. And qualitative results can be observed from the image itself.
\end{abstract}

\section{General Terms}

Digital Image Processing, Computer Vision

\section{Keywords}

Brightness Preservation, Image Enhancement, Bi-histogram Equalization, Tri-histogram Equalization

\section{INTRODUCTION}

Most of the consumer graded image sensing device capture images that are significantly flat. Thereby, contrast enhancement plays a major role in the improvement of visual quality in computer vision, pattern recognition and in the processing of digital images. Among many other techniques for contrast enhancement, Global Histogram Equalization (GHE) was the most extensively utilized one. The target of global histogram equalization is to achieve uniform distribution of intensities for any image, which is done by flattening the probability distribution of that image and stretching the dynamic range of gray levels. In theory, the mean brightness of the histogram equalized image is always the middle gray level regardless of the input mean, which in practice, makes this method less ideal for consumer electronic appliances where brightness preservation is a necessary aim [2].

Several algorithms have been proposed by many researchers over the recent years to solve the aforementioned problem of GHE. One of the earliest attempts was Brightness Preserving Bi-histogram equalization (BBHE) which divides the input image histogram into two parts based on the input mean brightness and equalize both parts individually to obtain the final image [4]. Later, [8] proposes a new method namely equal area Dualistic Sub-Image Histogram Equalization (DSIHE) in which the authors claimed to outperform BBHE in terms of brightness preservation and image content (entropy) preservation. In DSIHE, instead of using mean brightness to divide the histogram as in BBHE, median value was chosen.

Nevertheless, in cases where higher degree of brightness preservation is the requirement, it has been found that, both BBHE and DSIHE could not perform well. This leads to a number of other methods including Minimum Mean Brightness Error Bi-Histogram Equalization (MMBEBHE) [2], Recursive Mean-Separate Histogram Equalization (RMSHE) [1], Recursive Sub-Image Histogram Equalization (RSIHE) [6], Recursively Separated and Weighted Histogram Equalization (RSWHE) [3], etc. MMBEBHE provides a novel extension of bi-histogram equalization method where the histogram is divided into two parts by a threshold value which yields minimum difference between input mean and output mean. While other techniques like RMSHE, RSIHE and RSWHE divide the input histogram recursively. RMSHE uses mean brightness to divide the histogram while RSIHE uses median value instead. RSWHE is similar to RMSHE and RSIHE with an addition of a weighting process using normalized power law function.

Among some of the recent techniques, Exposure based Sub Image Histogram Equalization (ESIHE) [7] tries to separate the input histogram into two by finding an exposure threshold value and then clipping the histogram using clipping threshold, finally equalizing both of the clipped histogram separated by the exposure threshold individually. Another technique known as Statistic Separate Tri-Histogram Equalization (SSTHE) [5] was the first instance of the trihistogram based separation technique which couldn't outperform any of the aforementioned bi-histogram based equalization methods.

Another tri-histogram based SSTHE variant can be used as contrast enhancement method where separating the histogram into three parts by finding two division point can be obtained from minimum and maximum intensity occurrence of the pixel values in the input image.

The rest of the paper is organized as follows: Section 2 presents the study of bi-histogram based enhancement approach. Section 3 explain how tri-histogram based approach can modify the workflow. Section 4 describes about absolute mean brightness error measurement feature used to measure enhanced images brightness preservation and quality. Section 5 gives experimental results with section 6 concluding the paper. 


\section{BI-HISTOGRAM BASED ENHANCEMENT APPROACH}

Suppose $X=\{X(i, j)\}$ denotes an input image composed of $\mathrm{L}$ discrete gray levels, denoted by $\left\{X_{0}, X_{1}, \ldots, X_{L-1}\right\}$ and $X_{t}$ denotes a threshold intensity value on which the histogram is divided into two parts where $X_{t} \in\left\{X_{0}, X_{1}, \ldots, X_{L-1}\right\}$. This intensity value $X_{t}$ can be used to divide the input image into two sub-images $X_{l}$ and $X_{h}$ such that,

$$
X=X_{l} \cup X_{h}
$$

where

$$
X_{l}=\left\{X(i, j) \mid X(i, j) \leq X_{t}, \forall X(i, j) \in X\right\}
$$

and

$$
X_{h}=\left\{X(i, j) \mid X(i, j)>X_{t}, \forall X(i, j) \in X\right\}
$$

This means, sub-image $X_{l}$ consist of all pixels with intensity values in the range $\left\{X_{0}, X_{1}, \ldots, X_{t}\right\}$ and $X_{h}$ consist of all pixels with intensity values in the range $\left\{X_{t+1}, X_{t+2}, \ldots, X_{L-1}\right\}$.

Now, the probability density function (PDF) of the subimages $X_{l}, X_{h}$ can be defined as

$$
P D F_{l}\left(X_{k}\right)=\frac{n_{k}}{n_{l}}
$$

where $k=0,1, \ldots, t$, and

$$
P D F_{h}\left(X_{k}\right)=\frac{n_{k}}{n_{h}}
$$

where $k=t+1, \quad t+2, \ldots, \quad L-1$. Here, $n_{l}=\sum_{k=0}^{t} n_{k}$, and $n_{h}=\sum_{k=t+1}^{L-1} n_{k}$ represents the total number of pixels in the sub-image $X_{l}$, and $X_{h}$ respectively. Note that, the total number of pixels in the image is $N=n_{l}+n_{h}$, by definition. From the above definition of the probability density function, cumulative density function (CDF) can be defined as

$$
C D F_{l}\left(X_{k}\right)=\sum_{j=0}^{t} P D F_{l}\left(X_{j}\right)
$$

where $k=0,1, \ldots, \min$, and

$$
C D F_{h}\left(X_{k}\right)=\sum_{j=t+1}^{L-1} P D F_{h}\left(X_{j}\right)
$$

where $k=t+1, t+2, \ldots, L-1$. Note that, by definition, $C D F_{l}\left(X_{k}\right)=C D F_{h}\left(X_{k}\right)=1$.

We can define the transformation function $f_{l}\left(X_{k}\right)$, and $f_{h}\left(X_{k}\right)$ exploiting the cumulative density functions obtained from Eqs. (6) and (7) as

$$
f_{l}\left(X_{k}\right)=X_{0}+\left(X_{t}-X_{0}\right) C D F_{l}\left(X_{k}\right)
$$

and

$$
f_{h}\left(X_{k}\right)=X_{t+1}+\left(X_{L-1}-X_{t+1}\right) C D F_{h}\left(X_{k}\right)
$$

Based on these transform functions, the decomposed subimages are equalized independently and the union of these equalized sub-images form the final output image. Mathematically we can state this as, $\mathrm{Y}$ is the output image found by

$$
Y=\{Y(i, j)\}=f_{l}\left(X_{k}\right) \cup f_{h}\left(X_{k}\right)
$$

As (8) and (9) suggest, $f_{l}\left(X_{k}\right)$ equalizes the input image over the range $\left(X_{0}, X_{t}\right)$ and $f_{h}\left(X_{k}\right)$ equalizes it over the range $\left(X_{t+1}\right.$, $\left.X_{L-1}\right)$. As a consequence, (10) equalizes the input image $X$ over the entire dynamic range $\left(X_{0}, X_{L-1}\right)$, which is expected to result in better brightness preservation than the global histogram equalization.

\section{TRI-HISTOGRAM BASED

ENHANCEMENT APPROACH

In contrast to the bi-histogram equalization methods described in the previous section, tri-histogram based equalization methods chooses two intensity values to divide the histogram of the input image into three parts and then equalize each part individually.

Suppose $X=\{X(i, j)\}$ denotes an input image composed of $\mathrm{L}$ discrete gray levels, denoted by $\left\{X_{0}, X_{1}, \ldots, X_{L-1}\right\}$ and $X_{\text {min }}$ and $X_{\max }$ denotes two threshold intensity values that are used to divide the input histogram into three parts, such that, $X_{\min } \in\left\{X_{0}, X_{1}, \ldots, X_{L-1}\right\} \quad$ and $X_{\max } \in\left\{X_{0}, X_{1}, \ldots, X_{L-1}\right\}$. These two intensity values can be used to divide the input image into three sub-images $X_{l}, X_{m}$ and $X_{u}$ such that,

$$
X=X_{l} \cup X_{m} \cup X_{u}
$$

where

$$
\begin{gathered}
X_{l}=\left\{X(i, j) \mid X(i, j) \leq X_{\min }, \forall X(i, j) \in X\right\} \\
X_{m}=\left\{X(i, j) \mid X_{\min }<X(i, j) \leq X_{\max }, \forall X(i, j) \in X\right\}
\end{gathered}
$$

and

$$
X_{u}=\left\{X(i, j) \mid X(i, j)>X_{\max }, \forall X(i, j) \in X\right\}
$$

This means, sub-image $X_{l}$ consist of all pixels with intensity values in the range $\left\{X_{0}, X_{1}, \ldots, X_{\min }\right\}, X_{m}$ consist of all pixels with intensity values in the range $\left\{X_{\min +1}, X_{\min +2}, \ldots, X_{\max }\right\}$ and $X_{u}$ consist of all pixels with intensity values in the range $\left\{X_{\max +1}, X_{\max +2}, \ldots, X_{L-1}\right\}$.

Now, the probability density function (PDF) of the subimages $X_{l}, X_{m}, X_{u}$ can be defined as

$$
P D F_{l}\left(X_{k}\right)=\frac{n_{k}}{n_{l}}
$$

where $k=0,1, \ldots, \min$, and

$$
P D F_{m}\left(X_{k}\right)=\frac{n_{k}}{n_{m}}
$$

where $k=\min +1, \min +2, \ldots, \max$, and

$$
P D F_{u}\left(X_{k}\right)=\frac{n_{k}}{n_{u}}
$$


where $k=\max +1, \max +2, \ldots, L-1$. Here, $n_{l}=\sum_{k=0}^{\min } n_{k}$, $n_{m}=\sum_{k=\min +1}^{\max } n_{k}$ and $n_{u}=\sum_{k=\max +1}^{L-1} n_{k}$ represents the total number of pixels in the sub-image $X_{l}, X_{m}$ and $X_{u}$ respectively. Note that, the total number of pixels in the image is $N=n_{l}+n_{m}+n_{u}$, by definition. From the above definition of the probability density function, cumulative density function $(\mathrm{CDF})$ can be defined as

$$
C D F_{l}\left(X_{k}\right)=\sum_{j=0}^{\min } P D F_{l}\left(X_{j}\right)
$$

where $k=0,1, \ldots, \min$, and

$$
C D F_{m}\left(X_{k}\right)=\sum_{j=\min +1}^{\max } P D F_{m}\left(X_{j}\right)
$$

where $k=\min +1, \min +2, \ldots, \max$, and

$$
C D F_{u}\left(X_{k}\right)=\sum_{j=\max +1}^{L-1} P D F_{u}\left(X_{j}\right)
$$

where $k=\max +1, \max +2, \ldots, L-1$. Note that, by definition, $C D F_{l}\left(X_{k}\right)=C D F_{m}\left(X_{k}\right)=C D F_{u}\left(X_{k}\right)=1$.

We can define the transformation function $f_{l}\left(X_{k}\right), f_{m}\left(X_{k}\right)$ and $f_{u}\left(X_{k}\right)$ exploiting the cumulative density functions obtained from Eqs. (8), (9) and (10) as

$$
\begin{gathered}
f_{l}\left(X_{k}\right)=X_{0}+\left(X_{\min }-X_{0}\right) C D F_{l}\left(X_{k}\right) \\
f_{m}\left(X_{k}\right)=X_{\min +1}+\left(X_{\max }-X_{\min +1}\right) C D F_{m}\left(X_{k}\right)
\end{gathered}
$$

and

$$
f_{u}\left(X_{k}\right)=X_{\max +1}+\left(X_{L-1}-X_{\max +1}\right) C D F_{u}\left(X_{k}\right)
$$

Based on these transform functions, the decomposed subimages are equalized independently and the union of these equalized sub-images form the final output image. Mathematically we can state this as, $\mathrm{Y}$ is the output image found by

$$
Y=\{Y(i, j)\}=f_{l}\left(X_{k}\right) \cup f_{m}\left(X_{k}\right) \cup f_{u}\left(X_{k}\right)
$$

As (21), (22) and (23) suggest, $f_{l}\left(X_{k}\right)$ equalizes the input image over the range $\left(X_{0}, X_{\min }\right), f_{m}\left(X_{k}\right)$ equalizes it over the range $\left(X_{\min +1}, X_{\max }\right)$ and $f_{u}\left(X_{k}\right)$ equalizes it over the range $\left(X_{\max +1}, X_{L-}\right.$ 1). As a consequence, (24) equalizes the input image $X$ over the entire dynamic range $\left(X_{0}, X_{L-1}\right)$, which is expected to result in better brightness preservation than the global histogram equalization.

\section{MEASUREMENT FEATURE TO ASSES IMAGE QUALITY}

In this section, we provide definition to the most extensively used image quality assessment feature to analyze the brightness preservation of an enhancement method, known as absolute mean brightness error (AMBE), which we used to analyze our test images.

\subsection{Absolute Mean Brightness Error (AMBE)}

Absolute mean brightness error is a measurement feature used to measure how close the mean brightness of the enhanced image is to the input image. Hence, it's a measure to prove the enhanced image's brightness preservation. AMBE is defined as

$$
\operatorname{AMBE}(X, Y)=\left|X_{m}-Y_{m}\right|
$$

where $X_{m}$ is the mean of the input image $\mathrm{X}=\{\mathrm{X}(\mathrm{i}, \mathrm{j})\}$ and $Y_{m}$ is the mean of the output enhanced image $Y=\{Y(i, j)\}$. Both of the mean brightness can be obtained by

$$
X_{m}=\frac{\sum_{K=1}^{L-1} K \cdot P D F(K)}{\sum_{K=1}^{L-1} P D F(K)}
$$

For a particular image, the smaller the value of AMBE, the better its brightness preservation is obtained.

\section{RESULTS AND DISCUSSIONS}

In this section, the simulation results are compared with the global histogram equalization method (GHE), along with some other bi-histogram and tri-histogram equalization techniques such as BBHE, DSIHE, MMBEBHE, ESIHE and SSTHE and SSTHE-variant. Eight different test images such as: Mount Teide, Fish, Wheel, House, Snow Hill, Cameraman, Restaurant and Sandwick are compared between different methods, while first three of which are presented for visual quality analysis.

Table 1 shows a matrix of AMBE measurements between test images where rows correspond to the test images and columns correspond to enhancement methods. As can be seen from table 1, compared to other methods, MMBEBHE performs significantly better than any other bi-histogram based methods and SSITHE-Variant performs better than other tri-histogram based methods. Although the later method is working well for the test images, it has been seen by comparing another set of 500 images that, on an average, MMBEBHE works better than SSITHE-Variant. MMBEBHE is also very good in terms of other measurement features such as PSNR, entropy and others, while other bi-histogram based methods such as BBHE, DSIHE, ESIHE, along with the existing tri-histogram based method plain SSTHE performs poorly in the tested cases. 
Table 1. AMBE measurement between test images

\begin{tabular}{|l|l|l|l|l|l|l|l|}
\hline \multirow{2}{*}{ File } & \multirow{2}{*}{ GHE } & \multicolumn{4}{|c|}{ Bi-HE Methods } & \multicolumn{2}{c|}{ Tri-HE Methods } \\
\cline { 3 - 8 } & & BBHE & DSIHE & MMBEBHE & ESIHE & SSTHE & SSTHE-var \\
\hline Mount Teide & 81.0916 & 27.8927 & 22.622 & 12.7794 & 56.5199 & 27.1936 & 11.8317 \\
\hline Fish & 42.5636 & 26.1404 & 25.0187 & 10.6076 & 18.8043 & 23.6254 & 4.2324 \\
\hline Wheel & 41.3501 & 10.3941 & 15.4912 & 1.3053 & 5.9665 & 16.9197 & 1.036 \\
\hline House & 52.658 & 16.4289 & 17.9424 & 14.7611 & 24.3205 & 15.1204 & 12.7702 \\
\hline Snow Hill & 3.3994 & 2.2087 & 2.2087 & 1.4095 & 10.8823 & 9.0521 & 1.119 \\
\hline Cameraman & 10.4783 & 24.2581 & 17.9147 & 1.1832 & 17.3724 & 21.091 & 0.68476 \\
\hline Restaurant & 20.561 & 10.0057 & 10.2009 & 1.2093 & 3.3959 & 11.1965 & 0.12589 \\
\hline Stanwick & 5.1929 & 5.3568 & 0.16319 & 9.8087 & 8.9367 & 14.0288 & 0.053192 \\
\hline
\end{tabular}

Qualitative measures are equally important along with quantitative measures, since contrast enhancement can only be appreciated if the resultant image gives a pleasing appearance. To test the robustness of the existing method, a range of low to high contrast images are used. All these images are analyzed using the existing bi-histogram and tri-histogram based methods.

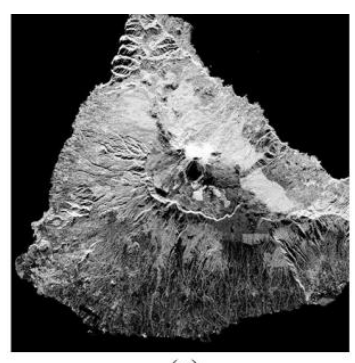

(a)

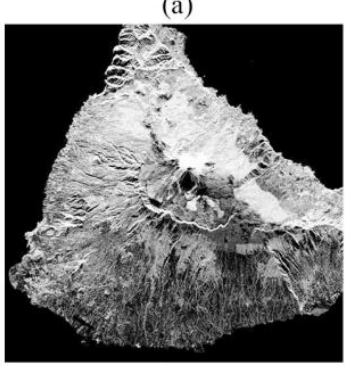

(e)

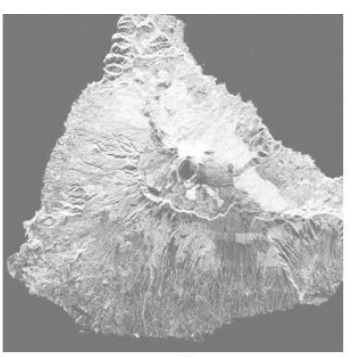

(b)

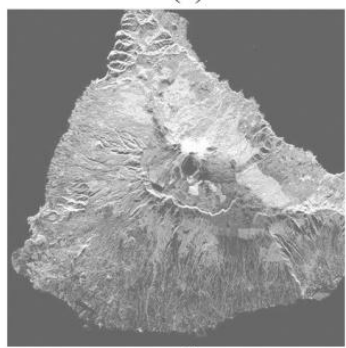

(f)

The concrete result of contrast enhancement is clearly observed in Figure 1 to 3 . If we closely observe the background of the Mount Teide image in Figure 1, we find that, GHE, BBHE, ESIHE, SSTHE significantly degrade the quality of that region by over-enhancement, while DSIHE, MMBEBHE and the SSITHE-Variant method provides better control for over-enhancement.

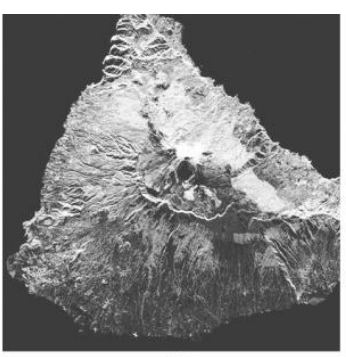

(c)

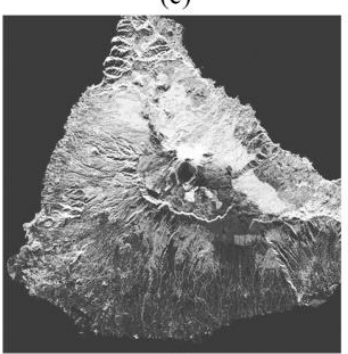

(g)

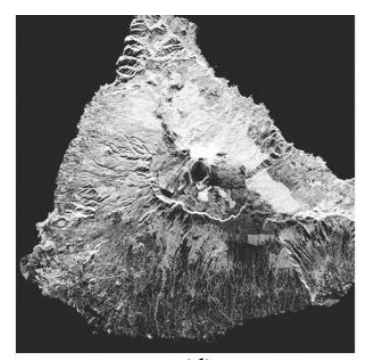

(d)

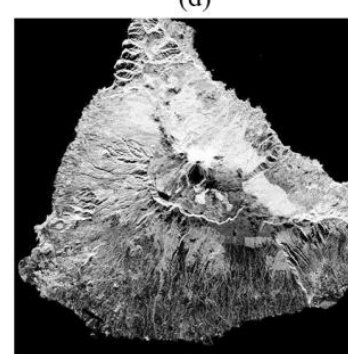

(h)

Figure 1. Enhancement results of Mount Teide image: (a) Original, (b) GHE, (c) BBHE, (d) DSIHE, (e) MMBEBHE, (f) ESIHE, (g) SSTHE, (h) SSTHE-Variant

The background of Figure 2 of the fish image is similarly over enhanced by GHE, BBHE, DSIHE, ESIHE and SSTHE methods. Only MMBEBHE method provides better overall enhancement. Again, Figure 3 shows that the jacket of the observer is blown out by GHE, BBHE, DSIHE and SSTHE, while rest of the methods performs quite well. Enhancement results for many other images also suggest that the MMITHE method provides most pleasing visual quality in most of the cases.

\section{CONCLUSION}

In this paper, we compared several contrast enhancement techniques based on bi-histogram and tri-histogram equalization. These methods works in a much better way when brightness preservation is of principle concern, compared to the global histogram equalization method. All these algorithms are also easy to implement in real-time processing. Also the enhanced images has a promising visual quality for display purpose in consumer graded electronic devices. 


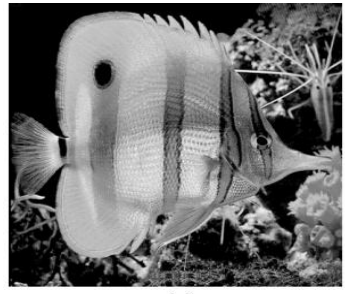

(a)

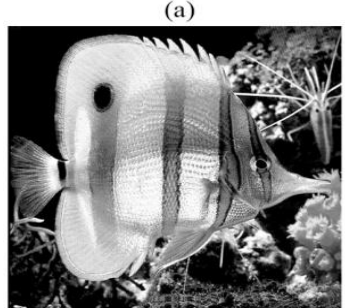

(e)

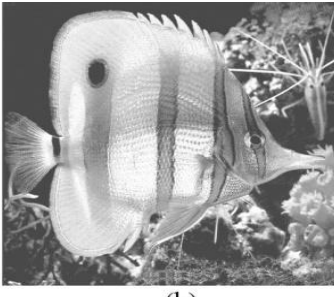

(b)

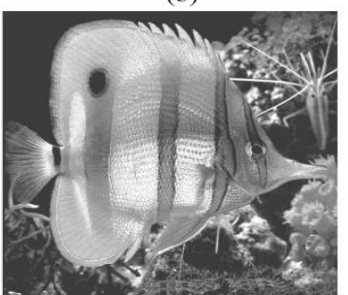

(f)

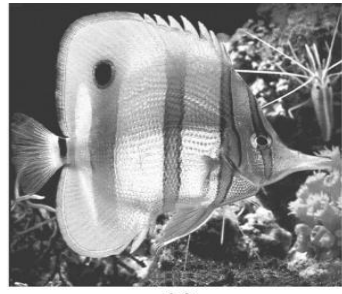

(c)

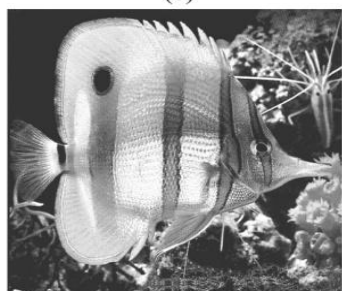

(g)
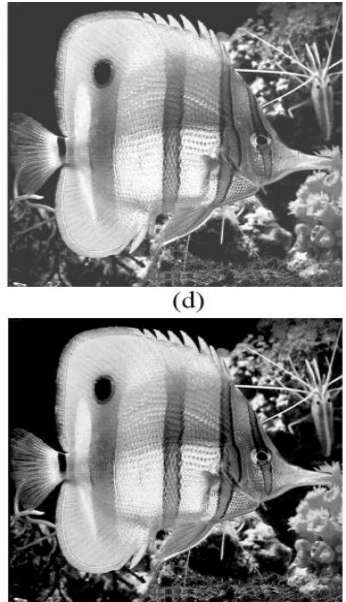

(h)

Figure 2. Enhancement results of Fish image: (a) Original, (b) GHE, (c) BBHE, (d) DSIHE, (e) MMBEBHE, (f) ESIHE, (g) SSTHE, (h) SSTHE-Variant

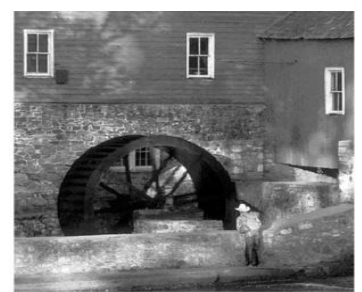

(a)

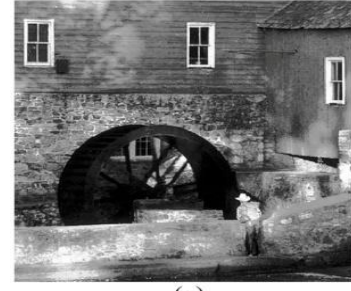

(e)

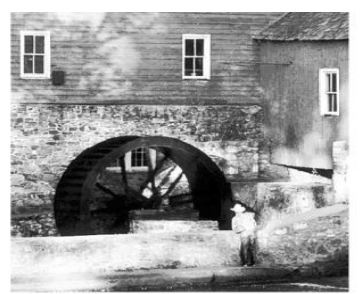

(b)

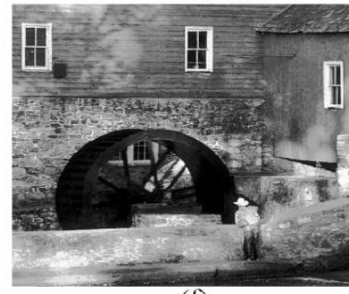

(f)

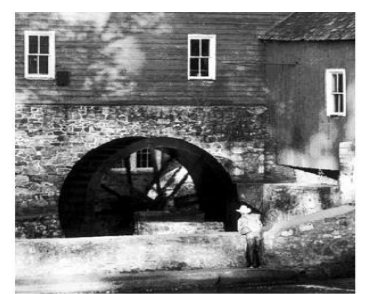

(c)

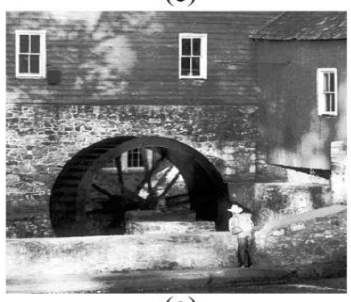

(g)

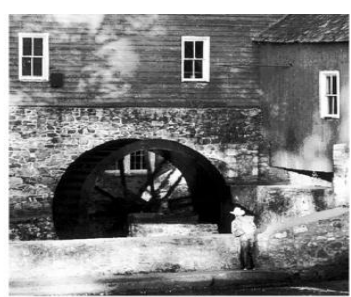

(d)

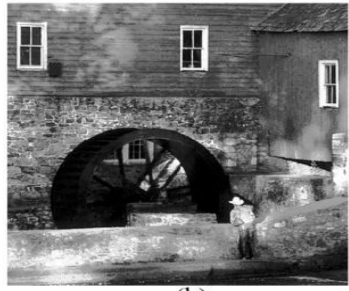

(h)

Figure 3. Enhancement results of Wheel image: (a) Original, (b) GHE, (c) BBHE, (d) DSIHE, (e) MMBEBHE, (f) ESIHE, (g) SSTHE, (h) SSTHE-Variant

\section{REFERENCES}

[1] Chen, S.D., Ramli, A.R., "Contrast enhancement using recursive mean-separate histogram equalization for scalable brightness preservation", IEEE Trans. on Consumer Electronics, vol. 49, no. 4, 2003, pp. 13011309.

[2] Chen, S.D., Ramli, A.R., "Minimum mean brightness error bi-histogram equalization in contrast enhancement”, IEEE Trans. Consumer Electron, vol. 49, no. 4, 2003, pp. 1310-1319.

[3] Kim, M., Chung, M.G., "Recursively separated and weighted histogram equalization for brightness preservation and contrast enhancement", IEEE Trans. Consumer Electron, vol. 54, no. 3, 2008, pp. 1389-1397.

[4] Kim, Y.T., "Contrast enhancement using brightness preserving bi-histogram equalization", IEEE Trans. on Consumer Electronics, vol. 43, no. 1, 1997, pp. 1-8.
[5] Lin, P.H., Lin, C.C., Yen, H.C., "Tri-Histogram Equalization Based on First Order Statistics", IEEE 13th International Symposium on Consumer Electronics, Kyoto, 2009, pp. 387-391.

[6] Sim, K.S., Tso, C.P., Tan, Y.Y., "Recursive sub-image histogram equalization applied to gray scale images", Pattern Recogn. Lett, vol. 28, no. 10, 2007, pp. 12091221.

[7] Singh, K., Kapoor, R., "Image enhancement using Exposure based Sub Image Histogram Equalization", Pattern Recogn. Lett, vol. 36, 2014, pp. 10-14.

[8] Wan, Y., Chen, Q., Zhang, B.M., "Image enhancement based on equal area dualistic sub-image histogram equalization method", IEEE Trans. Consumer Electron, vol. 45 , no. 1,1999 , pp. $68-75$. 